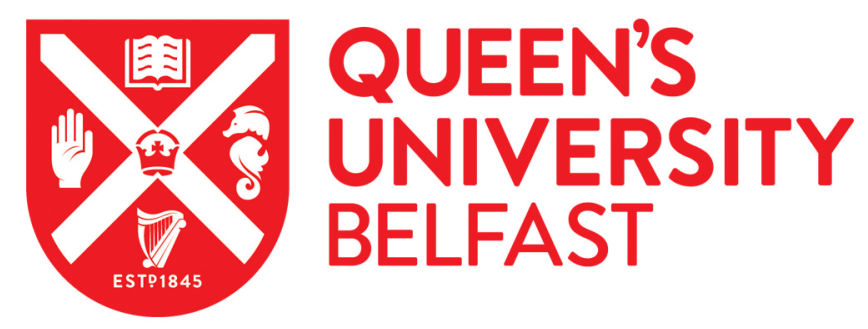

\title{
A Balancing Current Ratio based State-of-Health Estimation Solution for Lithium-ion Battery Pack
}

Tang, X., Gao, F., Liu, K., Liu, Q., \& Foley, A. M. (2021). A Balancing Current Ratio based State-of-Health Estimation Solution for Lithium-ion Battery Pack. IEEE Transactions on Industrial Electronics. https://doi.org/10.1109/TIE.2021.3108715

\section{Published in:}

IEEE Transactions on Industrial Electronics

\section{Document Version:}

Peer reviewed version

Queen's University Belfast - Research Portal:

Link to publication record in Queen's University Belfast Research Portal

\section{Publisher rights}

Copyright 2021, IEEE.

This work is made available online in accordance with the publisher's policies. Please refer to any applicable terms of use of the publisher.

\section{General rights}

Copyright for the publications made accessible via the Queen's University Belfast Research Portal is retained by the author(s) and / or other copyright owners and it is a condition of accessing these publications that users recognise and abide by the legal requirements associated with these rights.

Take down policy

The Research Portal is Queen's institutional repository that provides access to Queen's research output. Every effort has been made to ensure that content in the Research Portal does not infringe any person's rights, or applicable UK laws. If you discover content in the Research Portal that you believe breaches copyright or violates any law, please contact openaccess@qub.ac.uk. 


\title{
A Balancing Current Ratio based State-of-Health Estimation Solution for Lithium-ion Battery Pack
}

\author{
Xiaopeng Tang, Furong Gao, Kailong Liu, Member, IEEE, Qi Liu, and Aoife M. Foley, Senior Member, IEEE
}

\begin{abstract}
The inevitable battery ageing is a bottleneck that hinders the advancement of battery-based energy storage systems. Developing a feasible health assessment strategy for battery pack is important but challenging due to the joint requirements of the computational burden, modelling cost, estimation accuracy, and battery equalisation. This paper proposes a balancing current ratio (BCR)based solution to achieve reliable state-of-health (SoH) estimations of all series-connected cells within a pack while significantly reduce the overall reliance on cell-level battery models. Specifically, after employing BCR to describe the properties of the balancing process, the voltage-based active balancing is combined into the SoH estimator design for the first time, leading to a weighted fusion strategy to effectively estimate SoHs of all cells within a pack. Hardwarein-the-loop experiments show that even if a parameter-fixed open-circuit-voltage-resistance (OCV-R) model is used for modelling, the typical estimation error of our proposed solution can still be bounded by only $1.5 \%$, which is $70 \%$ lower than that of the benchmarking algorithms. Due to the model-free nature of the integrated voltage-based balancing, the robustness and flexibility of the proposed pack SoH estimation solution are also significantly improved.
\end{abstract}

Index Terms-Electric Vehicle, Lithium-ion Battery Pack, State-of-health Estimation, Balancing current ratio

\section{INTRODUCTION}

Lithium-ion (Li-ion) battery is regarded as a key energy storage source to promote the development of transportation electrification, with the overall capacity exceeding $170 \mathrm{GWh}$ in 2020 [1]. Though rechargeable, Li-ion batteries still suffer from inevitable ageing during their cyclic or even storage

Manuscript received 02-May-2021, revised 10-Jul-2021 and 06-Aug2021, accepted 18-Aug-2021.

This work was supported in part, by the Guangdong scientific and technological project (2017B010120002), Guangzhou Scientific and Technological Project (Grant No. 202002030323), Hong Kong Research Grants Council CERG project (16207717, 16208520), High Value Manufacturing (HVM) Catapult project under grant No 160080 CORE, and Shenzhen Science and Technology Innovation Commission under the grant Shenzhen-Hong Kong Innovation Circle Category D Project: SGDX 2019081623240948. (Corresponding author: Kailong Liu)

$X$. Tang and F. Gao are with the Department of Chemical and Biological Engineering, Hong Kong University of Science and Technology, Clear Water Bay, Kowloon, Hong Kong SAR, and F. Gao is also with Guangzhou HKUST Fok Ying Tung Research Institute, Guangzhou 511458, China (xtangai@connect.ust.hk; kefgao@ust.hk). K. Liu is with WMG, The University of Warwick, Coventry, CV4 7AL, UK (kailong.liu@warwick.ac.uk \& kliu02@qub.ac.uk) Q. Liu is with Department of Physics, City University of Hong Kong, Tat Chee Avenue, Kowloon, Hong Kong SAR (qiliu63@cityu.edu.hk). A. M. Foley is with the School of Mechanical and Aerospace Engineering, Queen's University Belfast, Belfast BT9 5AH, UK (a.foley@qub.ac.uk) mode [2], [3]. The aged batteries, in general, exhibit reduced performance in capacity, power, and reliability [4], [5]. Without precise information on the battery's degradation, users' anxieties on vehicle's driving range, transient performance, and safety will increase, posing challenges to the popularisation of transportation electrification [6]. In light of this, an effective approach to evaluate the battery's ageing status is imperative [7].

In general engineering applications, battery ageing status is commonly described by the state-of-health $(\mathrm{SoH})$, defined as the percentage of the actual battery capacity to its rated capacity [8], [9]. Although the rated capacity is commonly provided by the battery manufacturer, the actual capacity of the battery cannot be directly measured as it is difficult to fully charge or discharge electric vehicles in daily applications [10], [11]. Therefore, battery SoH is indirectly estimated from some available measurements for online applications. From the application point of view, the estimate can be categorised into two levels, namely, 1) cell-level and 2) pack-level.

There are multiple types of methods for estimating the $\mathrm{SoH}$ of a single battery [12]. One popular solution is developing a model that maps the ageing-related features to the $\mathrm{SoH}$ values and then uses this well-developed model for real-time $\mathrm{SoH}$ estimation. For example, the electrochemical features such as electrochemical impedance spectroscopy (EIS) [13] and geometry features like the vertical slope at the corner of constant-current (CC) charging curve [14] can be mapped to the SoH through Gaussian Process Regression (GPR) models. Similarly, the features extracted from the incremental capacity (IC) curves are also mapped to $\mathrm{SoH}$ through polynomial fittings [15], [16] or complicated data-driven networks [17]. Another popular solution is the state-of-charge (SoC)-based SoH estimation. Specifically, this solution relies on the normalised value of differential capacity over differential SoC $\left(\frac{\Delta Q}{\Delta \mathrm{SoC}} / Q_{\text {nom }}\right)$. As $\mathrm{SoC}$ itself is also an important internal state within the battery management system, joint $\mathrm{SoC}$ and $\mathrm{SoH}$ estimators with different strategies have been developed [4]. Given that $\Delta \mathrm{SoC}$ is in the denominator and could directly affect $\mathrm{SoH}$ estimation results, the high-performance parameteradaptive SoC estimators are preferable [18], [19].

When it comes to the pack-level, the situation becomes significantly different for battery health assessment. First, multiple cells need to be handled simultaneously, resulting in a significantly increased computational burden. In this context, complicated models or algorithms would be improper for all cell estimations within a pack [20]. Second, batteries within a pack usually suffer from cell-to-cell inconsistency. That is, the 
parameters of each cell could become different [21]. In such cases, equipping each cell with an accurate model would bring high modelling cost. Besides, adopting parameter-adaptive models for each cell would become computationally complex, while employing parameter-fixed models would inevitably reduce the accuracy of SoH estimation. Moreover, for pack applications, balancing is generally required to handle the inconsistency of cells. Noting that the output current and energy efficiency of battery balancing hardware are generally not measured in real applications due to the additional hardware cost and increase system complexity [22], [23], the accurate current measurements for each cell in the pack are not always available. The employment of the additional sensors would increase costs, while the lack of current measurement, on the other hand, would reduce the accuracy of the related SoH estimation. To handle these issues, multiple researches have been carried out. For examples, polynomial fittings are developed to estimate the $\mathrm{SoH}$ of each cell in a $\mathrm{LiFePO}_{4}$ battery pack through analysing the IC curves [24]. The method is accurate and computationally effective, but the influence of the balancing on the calculation of IC trajectory is not considered. Hua et al. considered the passive equalisation when estimating the $\mathrm{SoH}$ for pack applications [25], but the computational burden of their nonlinear multi time-scale framework for $\mathrm{SoH}$ estimation would be increased. Liu et al. used a simple parameter-fixed open-circuit-voltageresistance (OCV-R) model to estimate the $\mathrm{SoH}$ of a pack through a low computational $\mathrm{V}_{\text {min }}$ extended Kalman filter [26], at the cost of reducing the estimation accuracy. The 'leader-follower' strategy could be applied to balance the computational burden and accuracy of pack SoH estimation [27], where the states of the 'leader' are accurately determined, while those of the 'followers' would be calibrated based on the voltage difference to save computation. In this strategy, the estimator of the 'followers' would be sensitive to the voltage noise. Advanced machine-learning algorithms such as particle swarm optimisation-genetic algorithm [28] or support vector machine [29] could be applied to achieve high-fidelity pack SoH estimations, but the high computational burden still hinders their wider applications.

Based upon the above discussions, estimating the $\mathrm{SoH}$ for all cells within a battery pack is a key but challenging research topic, primarily caused by the difficulties in battery modelling. Specifically, providing each cell with an accurate model could significantly increase the offline modelling cost; using parameter-adaptive approaches could be computationally costly for large-scale pack applications; and using parameterfixed model for the entire pack would inevitable reduce the estimation accuracy, even if this solution does not require heavy modelling work or online calculations. In addition, the influence of the balancing hardware is also rarely considered in the relevant research works. To handle this bottleneck engineering issue, a new solution for $\mathrm{SoH}$ estimation based on parameter-fixed OCV-R model is proposed in this study. By introducing the converged controlling parameters of the modelfree voltage-based active balancing into the $\mathrm{SoH}$ estimator design, the reliance of the resulted estimator on the battery modelling can be significantly reduced, leading to better robustness and generalisation of cell $\mathrm{SoH}$ estimations within a pack. In this context, even using a parameter-fixed OCV$\mathrm{R}$ model (denoted as 'static model' in the remainder of this paper) could achieve satisfactory SoH estimation performance. To be specific, the main contributions of this study can be summarised as follows:

- The concept of BCR is introduced into the field of cell SoH estimations within a pack for the first time. The feasibility of using BCR for $\mathrm{SoH}$ estimation is analytically derived. A weighted fusion strategy based on the BCR information is further proposed for $\mathrm{SoH}$ estimation.

- When using a parameter-fixed OCV-R model to implement the proposed $\mathrm{SoH}$ estimation for each cell within the pack, the maximum error of designed SoH estimator can still be well-controlled within $1.5 \%$, which is $70 \%$ better than that of the conventional ones.

- By introducing the BCR into the estimator design, the reliance of the $\mathrm{SoH}$ estimator on the battery model can be significantly reduced, leading to better robustness and generalisation of cell SoH estimations within a pack.

The remainder of this paper is organised as follows: Section II introduces the related experimental platform. A brief description of the conventional SoC-based $\mathrm{SoH}$ estimation is given in Section III, followed by the detailed analysis of the proposed solution. Hardware-in-the-loop experiments and the result analysis are presented in Section IV. Section V summarises this paper.

\section{Description of Experimental Platform}

Since the controlling parameters of the active balancing are utilised in the design of the $\mathrm{SoH}$ estimator, the implementation of our algorithm has to rely on a specific hardware system. For the purpose of facilitating the following descriptions and also ensuring the repeatability of the experiments, the specifications of the experimental platform are introduced here.

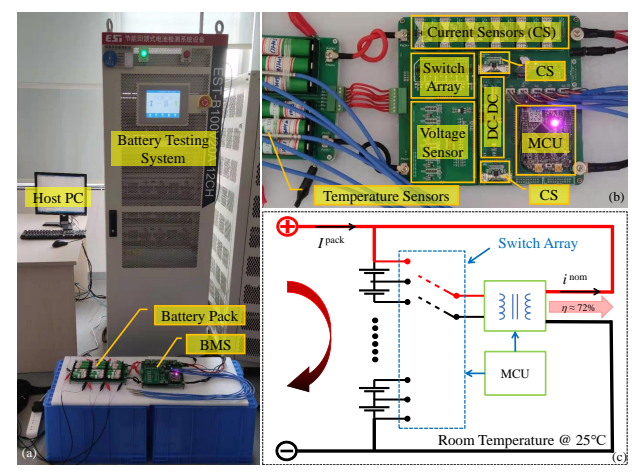

Fig. 1. Illustration of the experimental platform. (a): Photo of the overall experimental platform; (b): Photo of the utilised battery management system; and (c): simplified topology of the balancing circuit.

Fig. 1 illustrates our experimental platform. It contains a host PC, a battery testing system (BTS), a battery pack, and a battery management system (BMS). In our experiment design, the BTS is utilised to generate the driving current of the batteries, and the BMS is applied to implement the 
algorithms/functions such as sensing/estimation, active balancing, and communication. Some key data, such as the voltage, current, and efficiency, are sent back to the host PC for further analysis. Specifically, the BTS is produced by Guangdong East Star Technology Co., Ltd with an operating range of $0 \sim 100 \mathrm{~V}$ and $-20 \sim+20$ A. The battery pack contains 18 SONY VTC5 batteries with different ageing degrees, whose rated capacity is $2.5 \mathrm{Ah}$. The experimental methods for obtaining these aged cells are detailed in [30]. These batteries are connected with a 6-series-3-parallel (6S3P) configuration. Following the general engineering practice [31], each parallel group would be treated as a 'big cell' with the nominal capacity of 7.5Ah in the following study, unless otherwise specified. The actual capacities of these batteries are listed in Table I.

The real-time measurement and control of the batteries are implemented by our BMS. In this BMS, the DC-DC converter for active balancing is implemented with the LT8584 controller and NA6252 transformer. Its nominal output current is $-2.7 \mathrm{~A}$, while the typical efficiency $\eta$ is $72 \%$, counting in the ohmic loss on connectors, wires, and additional current sensors. In this way, the typical balancing current of the battery being operated is $-2.376 \mathrm{~A}$, while the value for the remaining batteries is $0.324 \mathrm{~A}^{1}$. The voltage measurement is implemented by the LTC6810 sensor with an equivalent 14-bit analogue-todigital converter (ADC), whose total maximum error under $25{ }^{\circ} \mathrm{C}$ can be limited within $1.8 \mathrm{mV}$. The pack current and the balancing current are measured by INA260 current-meters, which is a shunt-based $-15 \sim+15 \mathrm{~A}$ sensor with guaranteed accuracy of $0.15 \%$ under $25^{\circ} \mathrm{C}$. The resistance introduced by each sensor is $4.5 \mathrm{~m} \Omega$. Here, the balancing current is experimentally measured to facilitate the calculating of the 'referenced SoC' of the single batteries. Typical values of the balancing current $(-2.376 \mathrm{~A}$ and $0.324 \mathrm{~A})$ are used when implementing the proposed algorithm to simulate the reallife scenarios where balancing currents are not measured. The temperature is measured by the LM35D sensors, whose accuracy is $0.5^{\circ} \mathrm{C}$. All the above sensors are calibrated by an Agilent 34401A digital multimeter, whose accuracy can achieve $6 \frac{1}{2}$ bits. All experiments are carried out under a stable room temperature of $25^{\circ} \mathrm{C}$.

TABLE I

Capacities of the Selected batTeries (IN AH).

\begin{tabular}{lllllll}
\hline \hline No. & 1 & 2 & 3 & 4 & 5 & 6 \\
\hline Actual capacity & 6.524 & 6.855 & 6.873 & 7.450 & 7.382 & 7.215 \\
\hline \hline
\end{tabular}

The operating mode of our hardware platform is illustrated in Fig. 2. Here, $\Delta T=1000 \mathrm{~ms}$ is set as the sampling time and controlling interval, and the balancing hardware (if not halted) will work for approximately $\Delta t=950 \mathrm{~ms}$ in each operating cycle, leaving $50 \mathrm{~ms}$ to stabilise the terminal voltage, sampling, calculations, and necessary communications of the batteries.

\section{TECHNIQUE}

This section details our proposed solution for effective cell SoH estimations within a pack. The basic concepts and

${ }^{1}$ Cell voltages of a well-balanced pack are assumed to be similar.

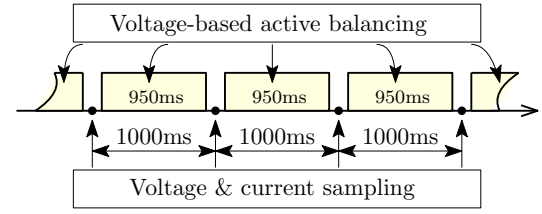

Fig. 2. Illustration of the hardware operations.

definitions are firstly introduced, followed by the descriptions of the conventional SoC-based SoH estimation approach. In Section III-C, the proposed solution is detailed. Benchmarking algorithms are finally introduced for comparison purposes.

\section{A. Concepts and definitions}

As our method enhances the conventional SoC-based $\mathrm{SoH}$ estimator with the BCR extracted from the battery equalisation process, to facilitate the following discussions, the related concepts are introduced in this subsection.

1) State-of-charge: For series-connected batteries within a pack, the SoC of cell $j$ at the sampling step $k$ is defined by:

$$
\operatorname{SoC}_{j}(k)=\operatorname{SoC}_{j}(0)+\sum_{l=0}^{l=k} \frac{\left[I^{\mathrm{pack}}(l) \cdot \Delta T+i_{j}^{\mathrm{bal}}(l) \cdot \Delta t\right]}{Q_{j}}
$$

where $\mathrm{SoC}_{j}(0)$ means the cell's initial SoC, $I^{\text {pack }}$ stands for the pack current introduced by the external loads as illustrated in Fig. 1, and $i^{\text {bal }}$ represents the balancing current. These two currents are defined to be positive if they tend to charge the battery cell. $Q_{j}$ is the cell's actual capacity.

2) State-of-health: For cell $j$, its SoH is defined as the ratio between $Q_{j}$ and the nominal capacity $Q_{\text {nom }}$ provided in the battery datasheet. Consequently, there exists:

$$
\mathrm{SoH}_{j}=Q_{j} / Q_{\mathrm{nom}}
$$

3) Batches: Before defining batches, the operating state of a battery pack is distinguished by an approximated averaging current derived from the low-pass filter:

$$
I_{\mathrm{avg}}(\alpha, k)=\alpha \cdot I_{\mathrm{avg}}(\alpha, k-1)+(1-\alpha) \cdot I^{\mathrm{pack}}(k)
$$

where $\alpha \in(0,1)$ is the filtering factor to compensate for the influence of dynamic load profiles. Here, the value of $\alpha$ is suggested to be 0.995 . Then, the operating mode of the battery pack can be defined as:

- Refuelling mode,

- Working mode

if $I_{\mathrm{avg}}(\alpha, k)>\delta$

- Idling mode,

if $I_{\mathrm{avg}}(\alpha, k)<-\delta$

if $\quad-\delta \leq I_{\text {avg }}(\alpha, k) \leq \delta$

where $\delta$ is a small positive real number used to compensate the uncertainties caused by current sensor drifting and noise, whose value is $0.1 \mathrm{~A}$ in this paper. With the well-defined operating modes, a batch can be defined based on the shifting of the operating modes [22]. To be specific, the refuelling batch is defined as a time period, whose starting point is defined as the time when the battery's operating mode switches from the other modes into the refuelling mode, and its end point is defined as the time when the operating mode switches from the refuelling mode into the other modes. Similarly, the working batch is defined as a time period, whose starting point 
is defined as the time when the operating mode switches from the other modes into the working mode, and its end point is defined as the time when the operating mode switches from the working mode into the other modes. The idling batch, again, is defined as a time period, whose starting point is defined as the time when the operating mode switches from the other modes into the idling mode, and its end point is defined as the time when the operating mode switches from the idling mode into the other modes. It is worth mentioning that these definitions are general and suitable for both constant-current and dynamic profiles.

4) Balancing current ratio $(B C R)$ : $\mathrm{BCR}$ reflects the ratio of the average balancing current to the average pack current [22]. For cell $j$, its BCR at time step $k$ is defined as:

$$
\mathrm{BCR}_{j}(k)=\left(\sum_{l=0}^{k} i_{j}^{\mathrm{bal}}(l) \cdot \Delta t\right) /\left(\sum_{l=0}^{k} I^{\mathrm{pack}}(l) \cdot \Delta T\right)
$$

Some comments need to be considered for this definition as:

1) The definition of BCR is only valid for refuelling and working batches. BCR is not defined for the idling batches because the denominator is generally zero or very close to zero.

2) The BCR is usually calculated within only one batch, even though this definition might work for multiple batches from the mathematical point of view. For instance, when considering a process with a working batch followed by a refuelling batch, two BCRs for these two batches are preferred to be defined, respectively.

3) Although the balancing current is not measured in most commercial BMSs, a nominal balancing current suggested by the manufacturer can be used to approximately calculate (1) and (4), at the cost of slightly sacrificing the estimation accuracy.

\section{B. Conventional algorithms}

The proposed method is established upon the conventional $\mathrm{SoH}$ estimation algorithms. For research completeness, a widely used conventional SoC-based $\mathrm{SoH}$ estimation solution is detailed here.

From the definitions in (1) and (2), one of the most simple and straightforward method for $\mathrm{SoH}$ estimation is to inversely calculate the actual capacity $Q_{j}$ based on accurate SoC estimation and capacity measurement from time $k_{1}$ to $k_{2}$, and then normalise this value with the nominal capacity $Q_{\text {nom }}$ as:

$$
\mathrm{SoH}_{j}^{k_{1} \rightarrow k_{2}}=\frac{\sum_{l=k_{1}}^{k_{2}}\left[I^{\mathrm{pack}}(l) \Delta T+i_{j}^{\mathrm{bal}}(l) \Delta t\right]}{\operatorname{SoC}_{j}\left(k_{2}\right)-\operatorname{SoC}_{j}\left(k_{1}\right)} / Q_{\text {nom }}
$$

Obviously, to implement this $\mathrm{SoH}$ estimator, online SoC estimations for single cells are required. Here, an SoC estimator based on the typical extended Kalman filters summarised in Algorithm 1, in which a simplified OCV-R model is adopted to describe the battery's dynamic as [32]:

$$
\begin{aligned}
\operatorname{SoC}_{j}(l) & =\operatorname{SoC}_{j}(l-1)+\left(I^{\mathrm{pack}}(l) \Delta T+i_{j}^{\mathrm{bal}}(l) \Delta t\right) / Q_{j} \\
V_{j}(l) & =f\left(\operatorname{SoC}_{j}(l), I^{\mathrm{pack}}(l)\right)=V_{o c}\left(\operatorname{SoC}_{j}(l)\right)+I^{\mathrm{pack}}(l) \cdot R_{j} \\
& =\sum_{n=0}^{5}\left\{a_{j, n} \cdot\left[\operatorname{SoC}_{j}(l)\right]^{n}\right\}+I^{\mathrm{pack}}(l) \cdot R_{j}
\end{aligned}
$$

where $a_{j, n}$ with $n \in[0,5]$ are the model parameters to be identified for cell $j, R_{j}$ is the ohmic resistance of cell $j$, which should also be determined in advance, $V_{j}$ means the terminal voltage of battery $j$, and $V_{o c}(\cdot)$ is a function describing the OCV-SoC relationship of the batteries. The model's identification can be implemented with direct-leastsquare algorithm, readers may refer to our previous work Ref [33] for details.

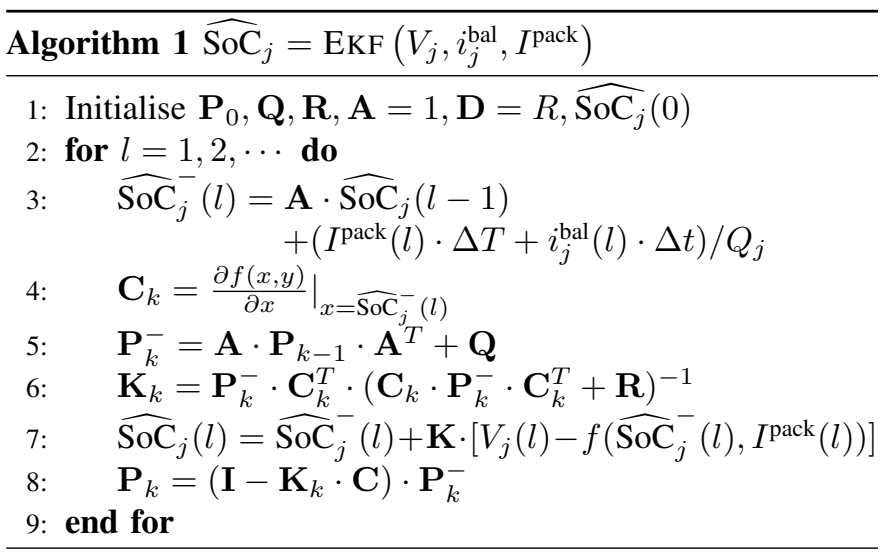

There are three reasons for selecting the OCV-R model in our study. First, the computational burden is low. The EKF algorithm with OCV-R model does not involve the calculation of matrix inverse $(\mathbf{P}, \mathbf{Q}, \mathbf{R}, \mathbf{A}, \mathbf{B}, \mathbf{C}, \mathbf{D}, \mathbf{K}$, and $\mathbf{I}$ are all scalar), making it more suitable for embedded applications. Second, when accurate model parameters are not available, complex models may not necessarily exhibits better accuracy. Lastly, with fewer parameters, the robustness and reliability of the OCV-R model is usually higher than that of the complex ones when model mismatch exists.

\section{Proposed BCR based solution}

With the well-defined SoC, SoH, BCR, and their conventional estimation schemes, this subsection details our designed solution. It should be known that when the ideal SoC-based balancing is applied, the SoC of each cell within a battery pack remains the same at any time step, and the maximum available capacity of this pack can be directly calculated by averaging the cell capacities. Therefore, when the pack SoC changes from $z_{1}$ to $z_{2}$ during the time $k_{1} \rightarrow k_{2}$, the capacity change of the pack can be calculated by:

$$
\Delta Q_{\mathrm{pack}}^{k_{1} \rightarrow k_{2}}=\sum_{l=k_{1}}^{k_{2}} I^{\mathrm{pack}}(l) \cdot \Delta T=\sum_{j=1}^{N} Q_{j} \cdot \frac{\left(z_{2}-z_{1}\right)}{N}
$$

For cell $j$, its capacity change from time $k_{1}$ to $k_{2}$ is caused by the joint effort of pack current and balancing current

$$
\begin{aligned}
\Delta Q_{j}^{k_{1} \rightarrow k_{2}} & =Q_{j} \cdot\left(z_{2}-z_{1}\right) \\
& =\sum_{l=k_{1}}^{k_{2}} I^{\mathrm{pack}}(l) \cdot \Delta T+\sum_{l=k_{1}}^{k_{2}} i_{j}^{\mathrm{bal}}(l) \cdot \Delta t
\end{aligned}
$$


The difference between $\Delta Q_{\mathrm{pack}}^{k_{1} \rightarrow k_{2}}$ and $\Delta Q_{j}^{k_{1} \rightarrow k_{2}}$ is caused by the additional active balancing:

$$
\Delta Q_{j}^{k_{1} \rightarrow k_{2}}-\Delta Q_{\mathrm{pack}}^{k_{1} \rightarrow k_{2}}=\sum_{l=k_{1}}^{k_{2}} i_{j}^{\mathrm{bal}}(l) \cdot \Delta t
$$

After substituting (7), (8), (9) and (2) into (4), the BCR of cell $j$ can be expressed by ${ }^{2}$ :

$$
\begin{aligned}
\mathrm{BCR}_{j} & =\frac{\sum_{l=k_{1}}^{k_{2}} i_{j}^{\mathrm{bal}}(l) \cdot \Delta t}{\sum_{l=k_{1}}^{k_{2}} I^{\mathrm{pack}}(l) \cdot \Delta T}=\frac{\Delta Q_{j}^{k_{1} \rightarrow k_{2}}-\Delta Q_{\mathrm{pack}}^{k_{1} \rightarrow k_{2}}}{\Delta Q_{\mathrm{pack}}^{k_{1} \rightarrow k_{2}}} \\
& =\frac{N \cdot Q_{j} \cdot\left(z_{2}-z_{1}\right)}{\left(Q_{1}+Q_{2}+\cdots+Q_{N}\right) \cdot\left(z_{2}-z_{1}\right)} \cdot \frac{\frac{1}{Q_{\text {nom }}}}{\frac{1}{Q_{\text {nom }}}}-1 \\
& =\left(N \cdot \mathrm{SoH}_{j}\right) /\left(\sum_{i=1}^{N} \mathrm{SoH}_{i}\right)-1
\end{aligned}
$$

Therefore, the relationship between batteries' SoH and BCR can be established as:

$$
\left\{\begin{array}{c}
\mathrm{BCR}_{1}=\left(N \cdot \mathrm{SoH}_{1}\right) /\left(\sum_{i=1}^{N} \mathrm{SoH}_{i}\right)-1 \\
\mathrm{BCR}_{2}=\left(N \cdot \mathrm{SoH}_{2}\right) /\left(\sum_{i=1}^{N} \mathrm{SoH}_{i}\right)-1 \\
\vdots \\
\mathrm{BCR}_{N}=\left(N \cdot \mathrm{SoH}_{N}\right) /\left(\sum_{i=1}^{N} \mathrm{SoH}_{i}\right)-1
\end{array}\right.
$$

There are $N$ sub-equations in (11), but the overall degreeof-freedom here is only $N-1$, implying that the $\mathrm{SoH}$ values cannot be directly observed from the measured BCR values [34]. However, this relationship can still be used for enhancing the cell $\mathrm{SOH}$ estimation performance within a pack.

We here denote the BCR calculated from (4) by using the nominal balancing current as $\widetilde{\mathrm{BCR}}$, and the SoH estimated from the conventional approach as $\widehat{\mathrm{SoH}}$. Then, with all BCR information $\widetilde{\mathrm{BCR}}_{1: N}$ and the one accurate SoH estimation $\widetilde{\mathrm{SoH}}_{j^{*}}$ available, the SoH of the remaining $N-1$ batteries in this pack could be readily calculated as:

$$
\widehat{\mathrm{SoH}}_{j}=\left(\widetilde{\mathrm{BCR}}_{j}+1\right) /\left({\widetilde{\mathrm{BCR}_{j}}}+1\right) \cdot{\widetilde{\mathrm{SoH}^{*}}}_{j^{*}}
$$

where $j \in[1, N], j^{*} \in[1, N]$.

From (12), the estimation accuracy of single-cell $\mathrm{SoH}$ is directly determined by the accuracy of the selected $\mathrm{SoH}$ estimation, $\widetilde{\mathrm{SoH}}_{j^{*}}$. As it is difficult to ensure all $\widetilde{\mathrm{SoH}}_{1: N}$ are accurate when a static battery model is applied for SoH estimation, an additional method that can pick the best estimate(s) is required. Noting that the $\mathrm{SoH}$ in this paper is calculated from the differential capacity over differential SoC from time $k_{1} \rightarrow k_{2}$, the quality of $\mathrm{SoH}$ estimation can be indirectly evaluated by checking the voltage accuracy within this period. For cell $j$, the residual of the voltage estimation is defined by:

$$
\mathcal{E}_{j}^{k_{1} \rightarrow k_{2}}=\sqrt{\frac{\sum_{l=k_{1}}^{k_{2}}\left\|V_{l}-f\left(\hat{x}_{l}, I_{j}(l)\right)\right\|_{2}^{2}}{k_{2}-k_{1}+1}}
$$

In our framework, a typical EKF-based algorithm is utilised for the $\mathrm{SoC}$ estimation. If the selected battery model accurately matches the actual system, the residual will be an approximated zero-mean Gaussian white noise series. Therefore, the

${ }^{2}$ With ideal balancing, the BCR value is only associated with $\mathrm{SoH}$ of the cells, and will not change with the time $k$. adaptiveness factor $\omega_{j}$ of the $j^{\text {th }}$ conventional SoH estimator can be defined from the following Gaussian function [35]:

$$
\omega_{j}=\frac{1}{\sqrt{2 \pi} \sigma} \exp \left\{-\frac{\left\|\mathcal{E}_{j}^{k_{1} \rightarrow k_{2}}\right\|_{2}^{2}}{2 \sigma^{2}}\right\}
$$

After normalising the adaptiveness factor by:

$$
\bar{\omega}_{j}=\omega_{j} /\left(\sum_{i=1}^{N} \omega_{i}\right)
$$

a set of weighting factors suggesting the confidence of the conventional SoH estimation can be obtained. In other words, we believe that the accuracy of $\widehat{\mathrm{SoH}}_{j}$ is likely to be higher than $\widetilde{\mathrm{SoH}}_{i}$ if $\bar{\omega}_{j}>\bar{\omega}_{i}$.

With the above weighting factors, the estimation of cell $\mathrm{SoH}$ within a pack can be derived by the following weighted fusion strategy as:

$$
\widehat{\mathbf{S o H}}=\left[\begin{array}{llll}
\widehat{\mathrm{SoH}}_{1} & \widehat{\mathrm{SoH}}_{2} & \cdots & \widehat{\mathrm{SoH}}_{N}
\end{array}\right]=\overline{\boldsymbol{\omega}} \cdot\left(\boldsymbol{\Lambda}_{\widetilde{\mathbf{S o H}}} \cdot \boldsymbol{\Gamma}_{\overparen{\mathbf{B C R}}}\right)
$$

where $\overline{\boldsymbol{\omega}}=\left[\begin{array}{llll}\bar{\omega}_{1} & \bar{\omega}_{2} & \cdots & \bar{\omega}_{N}\end{array}\right], \boldsymbol{\Lambda}_{\widetilde{\mathrm{SoH}}}=\operatorname{Diag}\left(\left[\widetilde{\mathrm{SoH}}_{1}, \widetilde{\mathrm{SoH}_{2}}\right.\right.$, $\left.\left.\cdots, \widetilde{\mathrm{SoH}}_{N}\right]\right)$, and

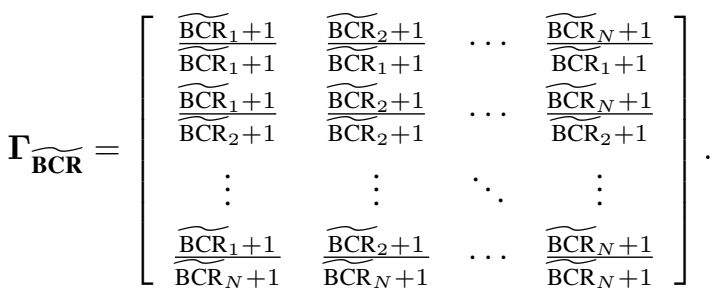

In this way, the accuracy of $\widehat{\mathrm{SoH}}$ will be determined by the best available $\widehat{\mathrm{SoH}}$ in this pack, while the large estimation error of the specific batteries caused by the mismatch of the static model can be alleviated.

It is worth noting that the above $\mathrm{SoH}$ estimation method (16) is established upon perfect SoC-based balancing. However, such balancing with accurate SoC information could be difficult to carry out. The main reasons are threefold: first, the balancing current and the efficiency of the converter are, in general cases, not measured. Only nominal values suggested by the manufacturer are available. Second, without reliable information on battery ageing, it is difficult to obtain high-fidelity SoC estimations. Third, giving each battery a parameter-adaptive model for SoC estimation, though theoretically appropriate, is still complex for real engineering applications. In summary, this strategy is not suitable for lowcost embedded microprocessors.

Given the reasons above, an alternative solution, voltagebased balancing, is selected to achieve efficient balancing. By defining $\mathbf{V}=\left\{V_{1}, \ldots, V_{N}\right\}$, the balancing procedure can be summarised in Algorithm 2.

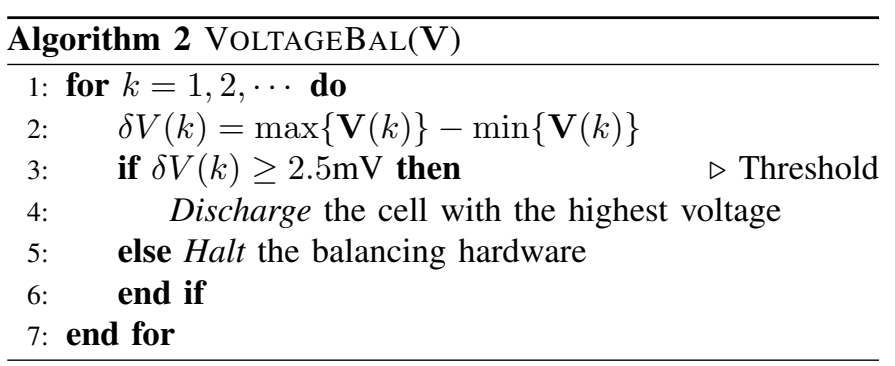


If the assumption 'batteries with the same SoC share the same terminal voltage' holds, the voltage-based balancing in Algorithm 2 is equivalent to the SoC-based balancing [22]. Since the key assumption here may be violated, the controlling accuracy of the voltage-based balancing would generally be inferior to that of the SoC-based balancing. However, it should be noted that the voltage-based balancing is model-free. Therefore, when using the converged controlling parameters (BCR in our case) to enhance the conventional $\mathrm{SoH}$ assessment, the new estimator can inherit the advantages of the model-free control, such as better reliability, enhanced robustness, and good generalisation performance.

It should also be pointed out that when using voltage-based balancing (especially for dynamic load profiles), 'repeated operations' may happen. That is, in the entire battery operating process, the hardware may discharge all cells and lead to some unnecessary and repeated operations. When the balancing hardware is 'perfect' with $100 \%$ energy efficiency, there will be no additional energy loss. However, when the influence of the energy loss cannot be neglected, it will affect our SoH estimation strategy as the summation of the BCR of all cells could be positive, rather than zero or close-to-zero. A quick calibration strategy here is to use the zero-mean $\mathrm{BCR}, \widehat{\mathrm{BCR}}$, to replace the $\widehat{\mathrm{BCR}}$ in $\Gamma_{\widetilde{\mathbf{B C R}}}$ of (16) as:

$$
\widehat{\mathrm{BCR}}_{j}(k)=\widetilde{\mathrm{BCR}}_{j}(k)-\frac{1}{N} \sum_{i=1}^{N} \widetilde{\mathrm{BCR}}_{i}(k)
$$

The resulted $\boldsymbol{\Gamma}_{\widehat{\mathbf{B C R}}}$ matrix could be defined as $\left[\boldsymbol{\Gamma}_{\widehat{\mathbf{B C R}}}(i, j)\right]=$ $\frac{\widehat{\mathrm{BCR}}_{j}+1}{\widehat{\mathrm{BCR}}_{i}+1}$ accordingly, where $\boldsymbol{\Gamma}_{\widehat{\mathbf{B C R}}}(i, j)$ represents the element in the $i^{\text {th }}$ row, $j^{\text {th }}$ column of the $\Gamma_{\widehat{\mathbf{B C R}}}$ matrix.

Based upon the above clarifications, the overall flow of our derived SoH estimation algorithm can be summarised in Fig. 3.

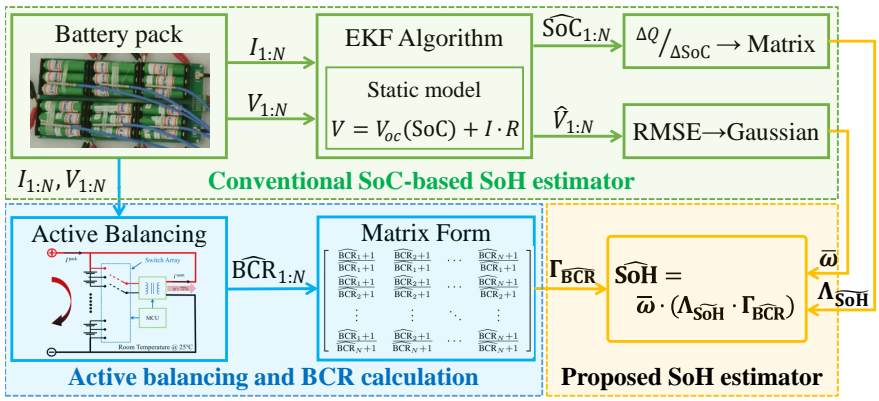

Fig. 3. The block diagram of the proposed method.

\section{Benchmarks and algorithm configurations}

For the purpose of comparing and highlighting the superiority of the proposed method, two typical benchmarking algorithms are introduced, together with the configurations of the proposed algorithm and the referenced $\mathrm{SoH}$.

1) Benchmark 1: Conventional SoH estimators with accurate models: For the first benchmarking algorithm, the conventional SoH estimators following (5) are applied. Here, the model parameters in (6) are identified respectively for each cell within a pack. These cells' capacities are set to be the actual value when estimating the SoC using Algorithm 1. With all modelling parameters available, benchmark 1 is expected to achieve the best performance under the selected SoH estimation framework. In the following text, 'AM' will be used to represent the 'accurate model' when necessary.

2) Benchmark 2: Conventional SoH estimators with static model: In the second benchmarking algorithm, the conventional SoH estimators following (5) are also applied. Here, it is assumed that the accurate model parameters for all batteries cannot be obtained, and a static model with fixed parameters is applied. To be specific, two cases are tested in this paper, where the model parameters in (6) are obtained from cell 1 and cell 4 in Table I, corresponding to the oldest and newest cell, respectively. The capacity of the static models is set to be the nominal one (7.5Ah here). In the remainder of this paper, 'SM1' will be used to denote the static model whose parameters are obtained from cell 1 , but the capacity is set to the nominal value. Similarly, 'SM4' will be used for cell 4.

3) Configurations of the proposed algorithm: In this case, the same configurations as that of benchmark 2 are utilised, and the BCR extracted from the balancing process will be used to enhance the conventional SoH estimations following (16).

4) Referenced values: The actual capacities of the selected 18 batteries are offline measured under $25^{\circ} \mathrm{C}$ with constantcurrent-constant-voltage (CCCV) charging and $\mathrm{CC}$ discharging profiles under $0.2 \mathrm{C}$ rate, with the cut-off conditions of $4.2 \mathrm{~V}, 2.75 \mathrm{~V}$, and $0.05 \mathrm{C}$. The capacities of the parallelconnected battery groups described in Table I are obtained by adding the capacities of related candidates together. The referenced SoC is obtained from the Ah-counting method with accurate sensors, while the referenced $\mathrm{SoH}$ is derived from (2).

\section{Results AND discussions}

This section presents the results and discussions. After examining the results of conventional SoC-based SoH estimation, the performance of our proposed $\mathrm{SoH}$ estimator is evaluated and compared with the benchmarking algorithms.

\section{A. State-of-charge estimations}

As the performance of the SoH estimators here highly relies on the related SoC estimations, this subsection first analyses the results of each cells' SoC estimation within the battery pack. For the EKF-based SoC estimation, $\mathbf{P}_{0}=0.25, \mathbf{Q}=$ $6.25 \cdot 10^{-6}$ and $\mathbf{R}=1$ are selected. The initial $\mathrm{SoC}$ is assumed to be known. Load profiles used for modelling and testing are detailed in Fig. 4-(a), respectively.

In this study, the EKF algorithms with the same parameter configurations but different models are used to estimate the SoC of each single cell. The comparisons between giving each cell an accurate model (AM, described in benchmark 1 of Section III-D) and giving all cells the same parameter-fixed model (SM1 and 4, described in benchmark 2 of Section III-D) are shown in Fig. 5, with the estimating errors given in Table II. As expected, the results corresponding to the accurate models are better than those with static models. When accurate 

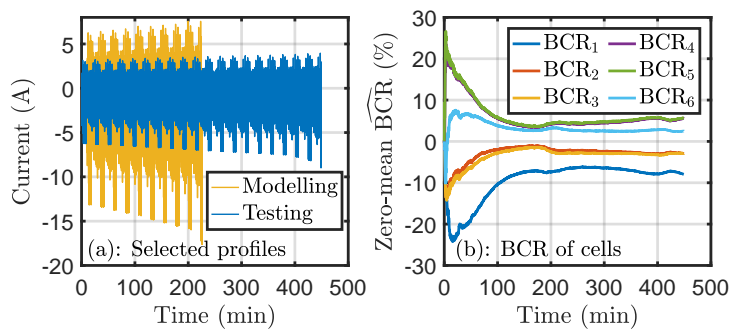

Fig. 4. Current and BCR. (a): Illustration of the current profiles for modelling and testing; (b): The estimated zero-mean BCR values of the six cells.

models are available, even though a low-computational OCV$\mathrm{R}$ model is applied, the RMSE of the SoC estimation can still be limited within $3 \%$. However, when it comes to cases with static models, the error would inevitably increase. In both cases (SM1 and SM4), the root-mean-square error (RMSE) of the estimations exceed $3 \%$. However, note that only a parameterfixed OCV-R model is available, and also the fact that the difference between the referenced and actual capacity of some batteries can exceed $10 \%$, the estimation presented here is still reasonably good.
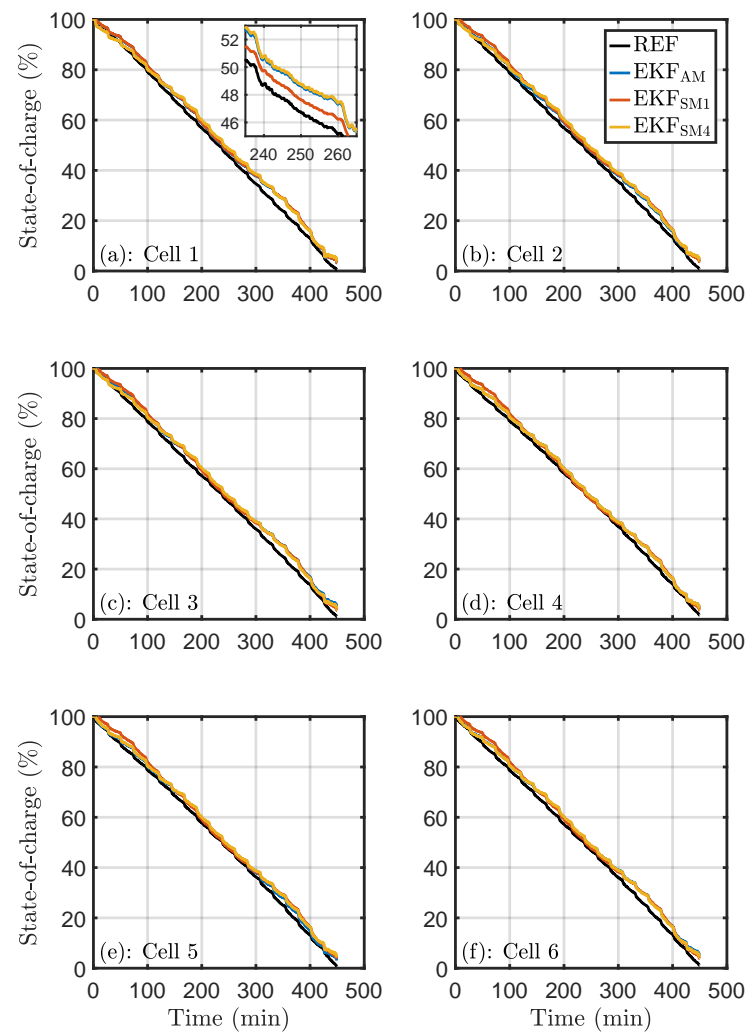

Fig. 5. Comparison of SoC estimation results. (a): results of cell 1; (b): results of cell 2; (c): results of cell 3 ; (d): results of cell 4; (e): results of cell 5 ; and (f): results of cell 6 . Here, 'REF' is short for 'referenced'.

\section{B. State-of-health estimations}

With each cell's SoC estimation available, the conventional and proposed $\mathrm{SoH}$ estimation solutions can be readily implemented on each single cell. To be specific, when calculating the $\mathrm{SoH}$ with (5), we start with a common case that
TABLE II

ROOT-MEAN-SQUARE-ERRORS OF THE SOC ESTIMATIONS (IN \%).

\begin{tabular}{lllllll}
\hline \hline No. & 1 & 2 & 3 & 4 & 5 & 6 \\
\hline EKF $_{\text {AM }}$ & 2.71 & 2.37 & 2.78 & 1.99 & 1.87 & 2.78 \\
EKF $_{\text {SM } 1}$ & 2.92 & 3.32 & 3.29 & 3.27 & 3.26 & 3.34 \\
EKF $_{\text {SM } 4}$ & 3.01 & 3.36 & 3.33 & 3.29 & 3.27 & 3.36 \\
\hline \hline
\end{tabular}

$\operatorname{SoC}_{j}\left(k_{1}\right)=\overline{\operatorname{SoC}}\left(k_{1}\right)=80 \%$ and $\operatorname{SoC}_{j}\left(k_{2}\right)=\overline{\operatorname{SoC}}\left(k_{2}\right)=$ $30 \%$, where $\overline{\mathrm{SoC}}$ stands for the average $\mathrm{SoC}$ of the cells in this pack. When calculating the adaptiveness factor in (14), $\sigma=\sqrt{\mathbf{Q}}=0.0025$ is selected.

In this section, the conventional SoC-based SoH estimator with SoC obtained from AM (benchmark 1), the conventional SoC-based SoH estimator with SoC obtained from SM1 and SM4 (benchmark 2), and the proposed method in which the SoC values are obtained from SM1 and SM4 are experimentally compared. The related $\mathrm{SoH}$ estimation results are shown in Fig. 6, while their errors are listed in Table III. Some interesting observations could be made: First, as expected, if accurate models for SoC estimation are available, the corresponding SoH estimation results could be highly accurate. As described in Table III, the maximum absolute error can be well-controlled within $1.5 \%$. However, when we do not have accurate models for SoC estimation and have to use a static model for all batteries, the resulted $\mathrm{SoH}$ error will significantly increase, exceeding $4 \%$. The accuracy of the proposed method is at least $70 \%$ better than that of the conventional benchmark 2 , and is competitive to that of the state-of-the-art algorithms with complex models or algorithms [4], [36]-[38]. It is interesting to see that even if the capacity of the two static models are all set to be the rated one when the model parameters are selected from the oldest cell (cell 1), the largest $\mathrm{SoH}$ estimation error lies in the newest cell (cell 4), and vice versa. This result implies that the static model can significantly affect the accuracy of SoH estimation. It is also worth noting that none of the $\mathrm{SoC}$ error approaches $4 \%$ in our test, but the largest error of the SoC-based $\mathrm{SoH}$ estimation could exceed this value. This result implies that the $\mathrm{SoC}$ estimation error would be amplified to $\mathrm{SoH}$ since the differential SoC is put in the denominator. Further, the complexity of our method is almost the same as that of the benchmarks. When tested with Matlab 2021a using a laptop equipped with Core i7-8550 CPU and 8G RAM, the operating time of the proposed method is 18.0704 seconds for all six cells for the entire load profile lasting for 26950 seconds, while the that of the benchmark 2 is 18.0679 seconds, the difference is lower than $0.1 \%$.

TABLE III

ERRORS OF THE SOH ESTIMATIONS (IN \%).

\begin{tabular}{lllllll}
\hline \hline No. & 1 & 2 & 3 & 4 & 5 & 6 \\
\hline Benchmark 1 & -1.03 & -0.63 & -1.01 & 1.17 & 1.13 & 0.70 \\
Benchmark 2 SM1 & -1.21 & 1.23 & 1.74 & 4.33 & 3.25 & 2.69 \\
Benchmark 2 SM4 & -4.03 & -1.84 & -1.31 & 1.15 & 0.07 & 0.48 \\
Proposed SM1 & -1.05 & -0.44 & 0.26 & 0.85 & -0.32 & -0.31 \\
Proposed SM4 & -0.84 & -0.22 & 0.48 & 1.08 & -0.09 & -0.08 \\
\hline \hline
\end{tabular}

When it comes to the proposed solution, it can be found 

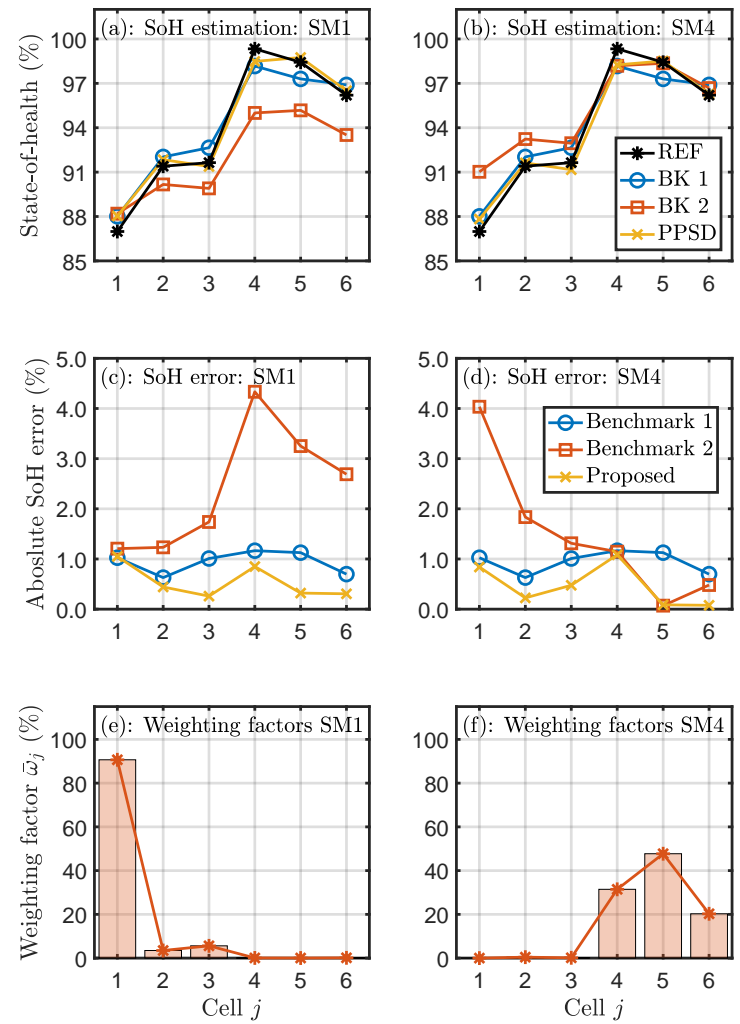

Fig. 6. SoH estimation results. (a): SoH estimation results with $\mathrm{SM} 1$ (b): SoH estimation results with SM4; (c): Absolute SoH estimation error with SM1; (d): Absolute SoH estimation error with SM4; (e): Weighting factors for fusion with SM1; (f): Weighting factors for fusion with SM4. Here, 'REF' is short for 'Referenced', 'BK 1' is short for 'benchmark 1', 'BK 2' is short for 'benchmark 2', and 'PPSD' is short for 'proposed'.

that the error of the SoH estimation can be well-controlled within $1.5 \%$, even if the error of the conventional approach (benchmark 2) can achieve 4\%. As described in (16), the value of $\widehat{\mathrm{SoH}}_{j}$ relies on the weighted combination of $\widehat{\mathrm{SoH}}_{1: N}$. Here, when SM1 is used, the weighting factor of cell 1, as illustrated in Fig. 6-(e), is the highest. When the 'SM4' is used in the proposed method, it is interesting to note that the weighting factor corresponding to batteries 5 and 6 are also high. From the posterior point of view, this result agrees that the accuracy of the conventional SoH estimations for cell 5 and 6 are also high, with errors lower than $0.5 \%$. For both two cases, as illustrated in Fig. 6-(e) and (f), the weighting factors corresponding to batteries with large $\mathrm{SoH}$ estimation error are negligible, and their side effects are therefore minimised. This result verifies the effectiveness of the proposed data fusion strategy in (16).

In addition, it is interesting to note that in Fig. 4-(b), the BCR values of the batteries can quickly converge to a stable value. As detailed in Table IV, with the joint influence of uncertain balancing efficiency, variable balancing current, and sub-optimal voltage-based balancing control, the difference between the zero-mean BCR (calculated from (17)) and the referenced BCR can still be bounded within 1.5\%. An accurate yet reliable BCR is the basis of using active balancing to calibrate the existing $\mathrm{SoH}$ estimations.

Noting that in the conventional SoH estimation framework
TABLE IV

BCR OF THE BATTERIES (IN \%).

\begin{tabular}{lllllll}
\hline \hline No. & 1 & 2 & 3 & 4 & 5 & 6 \\
\hline Referenced BCR & -7.46 & -2.76 & -2.51 & 5.68 & 4.71 & 2.34 \\
BCR from (17) & -6.51 & -2.47 & -2.96 & 4.59 & 4.87 & 2.49 \\
Error & -0.95 & -0.30 & 0.45 & 1.09 & -0.15 & -0.14 \\
\hline
\end{tabular}

described by (5), the values of $\overline{\mathrm{SoC}}\left(k_{1}\right)$ and $\overline{\mathrm{SoC}}\left(k_{2}\right)$ may also change with the specific applications. To further verify the generalisation of the proposed method, the influence of the two $\mathrm{SoC}$ values is examined by recording the maximum absolute SoH estimation error of the six cells. The results are given in Fig. 7.
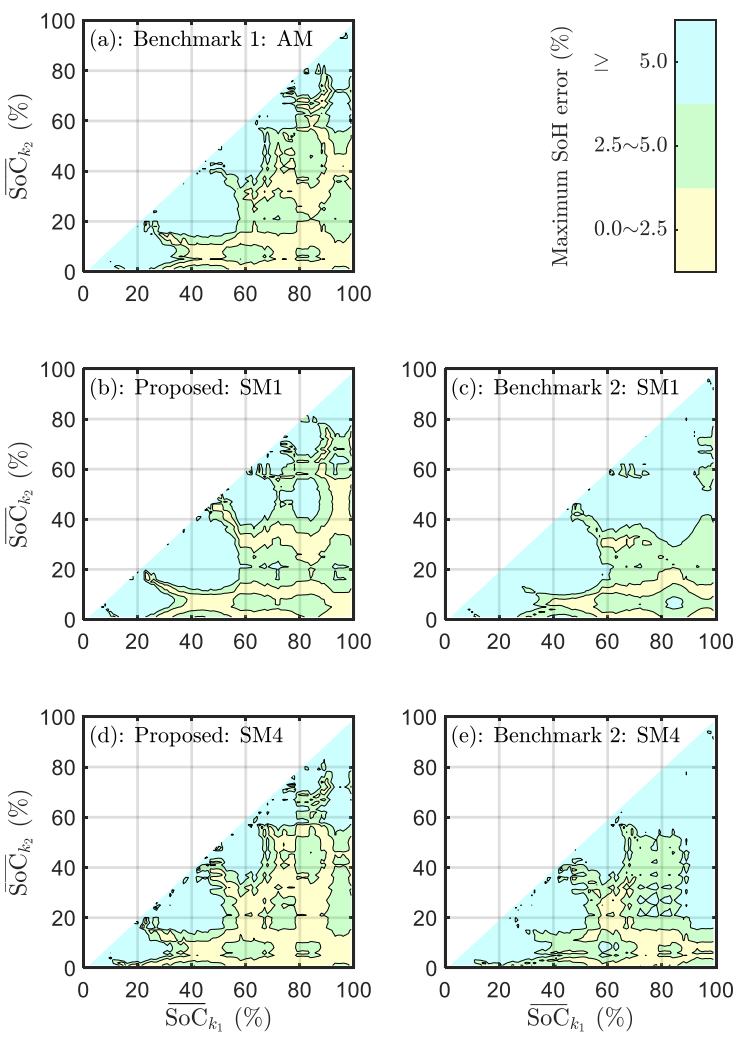

Fig. 7. The distribution of the maximum SoH estimation errors when differnet values of $\overline{\mathrm{SoC}}_{k_{1}}$ and $\overline{\mathrm{SoC}}_{k_{2}}$ are applied, where $\overline{\mathrm{SoC}}_{k_{1}}>\overline{\mathrm{SoC}}_{k_{2}}$. (a): Error distribution of benchmark 1 with AM. (b): Error distribution of the proposed method with SM1; (c): Error distribution of benchmark 2 with SM1; (d): Error distribution of the proposed method with SM4; and (e): Error distribution of benchmark 2 with SM4. Here, the area coloured with lightyellow represents that the corresponding maximum SoH error can be bounded by $2.5 \%$, while $5.0 \%$ for lightgreen, and $\geq 5.0 \%$ for the lightblue.

As illustrated in Fig. 7, the SoH estimation performance can change with the algorithm's specifications. Generally, when $\Delta$ SoC is smaller than $20 \%$, it is unlikely to obtain accurate SoH estimations. Further, by comparing the proposed method under the SM1 and SM4 situations (Fig. 7-(b) and (d)), it can be found that the selection of 'static model' can also influence the estimations. For instance, when the $\operatorname{SoC}\left(k_{2}\right)$ lies between 15\% 25\%, the proposed method with SM1 is obviously inferior to that with SM4. This result agrees with the fact described in Fig. 5 that the SoC estimation accuracy 
of cell 1 with SM1 is lower than that of cell 4 with SM4 in this specific range, with considerable local fluctuations. When comparing the proposed method with benchmark 2, our approach can obtain better results in more testing cases, implying that the proposed method is more robust than the conventional SoH estimators with static models. Actually, our performance is competitive to that of benchmark 1, where all model parameters are known. Compared with benchmark 1 where six models are required to achieve precise $\mathrm{SoH}$ estimations, the model reliance of the proposed method is reduced by $83 \%$ due to the utilisation of only one parameterfixed model.

\section{Further discussions}

The proposed method is highly extensible. By checking the deriving process of (16), it can be found that our method has no restrictions on the model type. If some higher accuracy but more complex models such as parameter-adaptive RC models can be used for SoC estimation, the overall accuracy of the proposed SoH method can be further improved. In addition, if a 'leader-follower' strategy is applied for the battery pack SoC estimation, so that the estimated SoC of a specific battery $j^{*}$ within the pack is more reliable than others. According to this prior knowledge, the weighting factor $\bar{\omega}$ can be determined. For example, by setting $\bar{\omega}_{j=j^{*}}=1$ and $\bar{\omega}_{j \neq j^{*}}=0$, the overall calculation can be further simplified to facilitate practical applications in cost-sensitive scenarios such as electric bikes or backup energy storage systems, where simple models and low-cost processors are more preferred. Besides, our method has no restrictions on the balancing hardware. The proposed method can work for both active and passive balancing if the cells' balancing currents could be real-time obtained. Further, with accurate $\mathrm{SoH}$ estimators, the lifetime prediction will also be considered as an interesting future research direction.

\section{CONCLUSION}

Designing reliable pack-level battery ageing assessment strategy is a key but challenging issue when considering the joint requirements of computational burden, modelling cost, estimation accuracy, and pack equalisation. This paper presents a balancing current ratio (BCR)-based solution that can effectively balance all these requirements when estimating the SoHs of all series-connected cells within a pack. To be specific, the concept of BCR is first introduced to describe the ratio of the average balancing current to the average pack current. Its relationship with $\mathrm{SoH}$ for the ideal balancing process is then strictly derived, on the basis of which a weighted model-fusion strategy is further developed to update the conventional SoH estimations with the approximated BCR extracted from voltage-based balancing processes. Hardwarein-the-loop experiments are carried out to verify the proposed method, and our SoH estimation error can be bounded by $1.5 \%$ when using only the parameter-fixed OCV-R model, which is $70 \%$ better than that of the benchmark solution. Compared with the conventional estimators, the reliance of the proposed solution on the cell-level battery models is reduced by at least $83 \%$ due to the integration of the model-free balancing control. Given the reduction in modelling cost and improvement in algorithm robustness, the proposed method can meet the requirement of general pack applications and provide good balance between the computational burden and estimation accuracy, paving way to the advancement of the cost-sensitive applications.

\section{REFERENCES}

[1] International Energy Agency, "Global EV outlook 2020." IEA Paris, 2020. [Online]. Available: https://www.iea.org/reports/ global-ev-outlook-2019

[2] Y. Wang, J. Tian, Z. Sun, L. Wang, R. Xu, M. Li, and Z. Chen, "A comprehensive review of battery modeling and state estimation approaches for advanced battery management systems," Renewable and Sustainable Energy Reviews, vol. 131, DOI 10.1016/j.rser.2020.110015, p. 110015 , Oct. 2020.

[3] G. Dong, W. Han, and Y. Wang, "Dynamic bayesian network based lithium-ion battery health prognosis for electric vehicles," IEEE Transactions on Industrial Electronics, DOI 10.1109/tie.2020.3034855, pp. $1-1,2020$.

[4] X. Hu, H. Yuan, C. Zou, Z. Li, and L. Zhang, "Co-estimation of state of charge and state of health for lithium-ion batteries based on fractionalorder calculus," IEEE Transactions on Vehicular Technology, vol. 67, DOI 10.1109/tvt.2018.2865664, no. 11, pp. 10319-10329, Nov. 2018.

[5] K. Liu, Y. Shang, Q. Ouyang, and D. Widanalage, "A data-driven approach with uncertainty quantification for predicting future capacities and remaining useful life of lithium-ion battery," IEEE Transactions on Industrial Electronics, DOI 10.1109/TIE.2020.2973876, pp. 1-1, 2020.

[6] Y. Liu, J. Li, Z. Chen, D. Qin, and Y. Zhang, "Research on a multiobjective hierarchical prediction energy management strategy for range extended fuel cell vehicles," Journal of Power Sources, vol. 429, DOI https://doi.org/10.1016/j.jpowsour.2019.04.118, pp. 55 - 66, 2019.

[7] X. Tang, K. Liu, K. Li, W. D. Widanage, E. Kendrick, and F. Gao, "Recovering large-scale battery aging dataset with machine learning," Patterns, DOI 10.1016/j.patter.2021.100302, p. 100302, Jun. 2021.

[8] Z. Wei, H. He, J. Pou, K.-L. Tsui, Z. Quan, and Y. Li, "Signaldisturbance interfacing elimination for unbiased model parameter identification of lithium-ion battery," IEEE Transactions on Industrial Informatics, DOI 10.1109/tii.2020.3047687, pp. 1-1, 2020.

[9] L. Cai, J. Meng, D.-I. Stroe, G. Luo, and R. Teodorescu, "An evolutionary framework for lithium-ion battery state of health estimation," Journal of Power Sources, vol. 412, DOI 10.1016/j.jpowsour.2018.12.001, pp. 615-622, Feb. 2019.

[10] S. Atalay, M. Sheikh, A. Mariani, Y. Merla, E. Bower, and W. D. Widanage, "Theory of battery ageing in a lithium-ion battery: Capacity fade, nonlinear ageing and lifetime prediction," Journal of Power Sources, vol. 478, DOI 10.1016/j.jpowsour.2020.229026, p. 229026, Dec. 2020.

[11] J. Wei, G. Dong, and Z. Chen, "Remaining useful life prediction and state of health diagnosis for lithium-ion batteries using particle filter and support vector regression," IEEE Transactions on Industrial Electronics, vol. 65, DOI 10.1109/tie.2017.2782224, no. 7, pp. 5634-5643, Jul. 2018.

[12] Y. Li, K. Liu, A. M. Foley, A. Z $\tilde{A}^{1 / 4} / \mathrm{ke}$, M. Berecibar, E. NaniniMaury, J. V. Mierlo, and H. E. Hoster, "Data-driven health estimation and lifetime prediction of lithium-ion batteries: A review," Renewable and Sustainable Energy Reviews, vol. 113, DOI https://doi.org/10.1016/j.rser.2019.109254, p. 109254, 2019.

[13] K. Goebel, B. Saha, A. Saxena, J. Celaya, and J. Christophersen, "Prognostics in battery health management," IEEE Instrumentation \& Measurement Magazine, vol. 11, DOI 10.1109/mim.2008.4579269, no. 4, pp. 33-40, Aug. 2008.

[14] D. Yang, X. Zhang, R. Pan, Y. Wang, and Z. Chen, "A novel gaussian process regression model for state-of-health estimation of lithium-ion battery using charging curve," Journal of Power Sources, vol. 384, DOI 10.1016/j.jpowsour.2018.03.015, pp. 387-395, Apr. 2018.

[15] J. Tian, R. Xiong, and Q. Yu, "Fractional-order model-based incremental capacity analysis for degradation state recognition of lithium-ion batteries," IEEE Transactions on Industrial Electronics, vol. 66, DOI 10.1109/tie.2018.2798606, no. 2, pp. 1576-1584, Feb. 2019.

[16] X. Tang, C. Zou, K. Yao, G. Chen, B. Liu, Z. He, and F. Gao, "A fast estimation algorithm for lithium-ion battery state of health," Journal of Power Sources, vol. 396, DOI https://doi.org/10.1016/j.jpowsour.2018.06.036, pp. 453 - 458, 2018. 
[17] X. Li, Z. Wang, L. Zhang, C. Zou, and D. D. Dorrell, "State-of-health estimation for li-ion batteries by combing the incremental capacity analysis method with grey relational analysis," Journal of Power Sources, vol. 410-411, DOI https://doi.org/10.1016/j.jpowsour.2018.10.069, pp. $106-114,2019$.

[18] C. Zhang, L. Y. Wang, X. Li, W. Chen, G. G. Yin, and J. Jiang, "Robust and adaptive estimation of state of charge for lithium-ion batteries," IEEE Transactions on Industrial Electronics, vol. 62, DOI 10.1109/tie.2015.2403796, no. 8, pp. 4948-4957, Aug. 2015.

[19] H. Chaoui, N. Golbon, I. Hmouz, R. Souissi, and S. Tahar, "Lyapunovbased adaptive state of charge and state of health estimation for lithiumion batteries," IEEE Transactions on Industrial Electronics, vol. 62, DOI 10.1109/tie.2014.2341576, no. 3, pp. 1610-1618, Mar. 2015.

[20] J. Meng, L. Cai, D.-I. Stroe, X. Huang, J. Peng, T. Liu, and R. Teodorescu, "An automatic weak learner formulation for lithium-ion battery state of health estimation," IEEE Transactions on Industrial Electronics, DOI 10.1109/tie.2021.3065594, pp. 1-1, 2021.

[21] K. Liu, Z. Yang, X. Tang, and W. Cao, "Automotive battery equalizers based on joint switched-capacitor and buck-boost converters," IEEE Transactions on Vehicular Technology, vol. 69, DOI 10.1109/tvt.2020.3019347, no. 11, pp. 12716-12 724, Nov. 2020.

[22] X. Tang, C. Zou, T. Wik, K. Yao, Y. Xia, Y. Wang, D. Yang, and F. Gao, "Run-to-run control for active balancing of lithium iron phosphate battery packs," IEEE Transactions on Power Electronics, vol. 35, DOI 10.1109/tpel.2019.2919709, no. 2, pp. 1499-1512, Feb. 2020.

[23] "LT8584 - 2.5A Monolithic Active Cell Balancer with Telemetry Interface." [Online]. Available: https://www.analog.com/media/en/ technical-documentation/data-sheets/8584fb.pdf

[24] P. Leijen, D. A. Steyn-Ross, and N. Kularatna, "Use of effective capacitance variation as a measure of state-of-health in a series-connected automotive battery pack," IEEE Transactions on Vehicular Technology, vol. 67, DOI 10.1109/tvt.2017.2733002, no. 3, pp. 1961-1968, Mar. 2018.

[25] Y. Hua, A. Cordoba-Arenas, N. Warner, and G. Rizzoni, "A multi time-scale state-of-charge and state-of-health estimation framework using nonlinear predictive filter for lithium-ion battery pack with passive balance control," Journal of Power Sources, vol. 280, DOI 10.1016/j.jpowsour.2015.01.112, pp. 293-312, Apr. 2015.

[26] X. Liu, Y. He, and Z. Chen, "State-of-charge estimation for power li-ion battery pack using v min-ekf," in The 2nd International Conference on Software Engineering and Data Mining, pp. 27-31. IEEE, 2010.

[27] H. Dai, X. Wei, Z. Sun, J. Wang, and W. Gu, "Online cell SOC estimation of li-ion battery packs using a dual time-scale kalman filtering for EV applications," Applied Energy, vol. 95, DOI 10.1016/j.apenergy.2012.02.044, pp. 227-237, Jul. 2012.

[28] X. Zhang, Y. Wang, C. Liu, and Z. Chen, "A novel approach of battery pack state of health estimation using artificial intelligence optimization algorithm," Journal of Power Sources, vol. 376, DOI 10.1016/j.jpowsour.2017.11.068, pp. 191-199, Feb. 2018.

[29] X. Feng, C. Weng, X. He, X. Han, L. Lu, D. Ren, and M. Ouyang, "Online state-of-health estimation for li-ion battery using partial charging segment based on support vector machine," IEEE Transactions on Vehicular Technology, vol. 68, DOI 10.1109/tvt.2019.2927120, no. 9, pp. 8583-8592, Sep. 2019.

[30] X. Tang, K. Liu, X. Wang, B. Liu, F. Gao, and W. D. Widanage, "Realtime aging trajectory prediction using a base model-oriented gradientcorrection particle filter for lithium-ion batteries," Journal of Power Sources, vol. 440, DOI 10.1016/j.jpowsour.2019.227118, p. 227118, Nov. 2019.

[31] R. Gogoana, M. B. Pinson, M. Z. Bazant, and S. E. Sarma, "Internal resistance matching for parallel-connected lithium-ion cells and impacts on battery pack cycle life," Journal of Power Sources, vol. 252, DOI 10.1016/j.jpowsour.2013.11.101, pp. 8-13, Apr. 2014.

[32] X. Hu, S. Li, and H. Peng, "A comparative study of equivalent circuit models for li-ion batteries," Journal of Power Sources, vol. 198, DOI 10.1016/j.jpowsour.2011.10.013, pp. 359-367, Jan. 2012.

[33] X. Tang, B. Liu, Z. Lv, and F. Gao, "Observer based battery SOC estimation: Using multi-gain-switching approach," Applied Energy, vol. 204, DOI 10.1016/j.apenergy.2017.03.079, pp. 1275-1283, Oct. 2017.

[34] D. G. Luenberger, "Observing the state of a linear system," IEEE Transactions on Military Electronics, vol. 8, DOI 10.1109/tme.1964.4323124, no. 2, pp. 74-80, 1964.

[35] C. Lin, H. Mu, R. Xiong, and W. Shen, "A novel multi-model probability battery state of charge estimation approach for electric vehicles using h-infinity algorithm," Applied Energy, vol. 166, DOI 10.1016/j.apenergy.2016.01.010, pp. 76-83, Mar. 2016.
[36] B. Jiang, H. Dai, X. Wei, and T. Xu, "Joint estimation of lithium-ion battery state of charge and capacity within an adaptive variable multitimescale framework considering current measurement offset," Applied Energy, vol. 253, DOI 10.1016/j.apenergy.2019.113619, p. 113619, Nov. 2019.

[37] B. Liu, X. Tang, and F. Gao, "Joint estimation of battery stateof-charge and state-of-health based on a simplified pseudotwo-dimensional model," Electrochimica Acta, vol. 344, DOI 10.1016/j.electacta.2020.136098, p. 136098, Jun. 2020.

[38] T. Ouyang, P. Xu, J. Lu, X. Hu, B. Liu, and N. Chen, "Co-estimation of state-of-charge and state-of-health for power batteries based on multithread dynamic optimization method," IEEE Transactions on Industrial Electronics, DOI 10.1109/tie.2021.3062266, pp. 1-1, 2021.

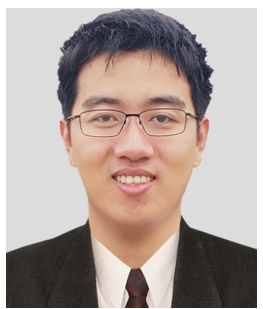

Xiaopeng Tang received the Ph.D. degree in chemical and biological engineering (CBE) with the Hong Kong University of Science and Technology (HKUST), Hong Kong, China, in 2020.

$\mathrm{He}$ is currently a postdoc researcher with the Dept. CBE, HKUST. His research interests include modeling and control of energy storage systems. He received a list of prestigious prizes including the Hong Kong PhD Fellowship Scheme and Hong Kong RGC Postdoctoral Fellowship Scheme.

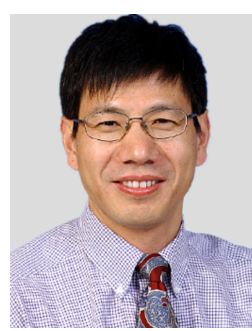

Furong Gao received the Ph.D. degrees in chemical engineering from McGill University, Montreal, QC, Canada in 1993.

$\mathrm{He}$ is currently a Chair Professor with the Department of Chemical and Biological Engineering, Hong Kong University of Science and Technology, Hong Kong, China. His research interests include process monitoring and fault diagnosis, batch process control, polymer processing control, and optimization. He is on editorial boards of a number of journals of his area.

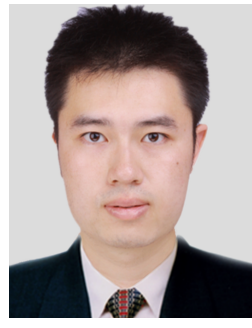

Kailong Liu (M'18) received the Ph.D. degree in electrical engineering from Queen's University Belfast, United Kingdom in 2018.

$\mathrm{He}$ is a Senior Research Fellow in the Warwick Manufacturing Group, University of Warwick, United Kingdom. His research interests include modeling, optimization and control with applications to electrical/hybrid vehicles, energy storage, battery manufacture and management. Dr. Liu is on editorial boards of some journals of his area including Renewable and Sustainable Energy Reviews, Control Engineering Practice.

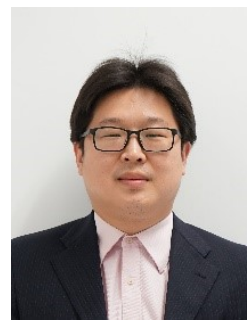

Qi Liu is currently an assistant professor in the Department of Physics, City University of Hong Kong. He obtained his Ph.D. from Purdue University in 2014.

Before joining CityU, he worked as a postdoctoral fellow at Argonne National Laboratory. His current research interests focus on the structureproperty studies of functional materials via multiple neutron- and synchrotron-based techniques. His broader research activities include the design and synthesis of novel energy storage materials, phase transition mechanisms and neutron-/synchrotron physics.

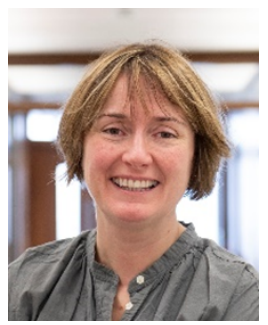

Aoife Foley (S'07-M'12-SM'21) received the B.E. (Civil Eng.) and her Ph.D. in unit commitment modelling of wind and energy storage in the Irish power system from University College Cork, Ireland, in 1996 and 2011, respectively.

Dr. Foley is currently a Reader with the School of Mechanical and Aerospace Engineering, Queen's University Belfast, Belfast, U.K. She is a Chartered Engineer (2001), a Fellow of Engineers Ireland since (2012), and a Senior Member of the IEEE (2021). She is also the Editor-in-Chief of Renewable and Sustainable Energy Reviews. 